PROCEEDINGS OF THE

AMERICAN MATHEMATICAL SOCIETY

Volume 131, Number 1, Pages 113-116

S 0002-9939(02)06536-X

Article electronically published on May 22, 2002

\title{
ON MODULES OF FINITE PROJECTIVE DIMENSION OVER COMPLETE INTERSECTIONS
}

\author{
S. P. DUTTA
}

(Communicated by Wolmer V. Vasconcelos)

\begin{abstract}
Recently Avramov and Miller proved that over a local complete intersection ring $(R, m, k)$ in characteristic $p>0$, a finitely generated module $M$ has finite projective dimension if for some $i>0$ and for some $n>0$, $\operatorname{Tor}_{i}^{R}\left(M, f_{R}^{n}\right)=0-f^{n}$ being the frobenius map repeated $n$ times. They used the notion of "complexity" and several related theorems. Here we offer a very simple proof of the above theorem without using "complexity" at all.
\end{abstract}

Recently Avramov and Miller $[\mathrm{A}-\mathrm{M}]$ gave an important characterization for modules of finite projective dimension over complete intersections in positive characteristic. To describe their result we need the following set-up:

Let $(R, m)$ be a local ring of characteristic $p>0$ with residue field $k=R / m$. Let $f: R \rightarrow R, f(x)=x^{p}$, denote the frobenius map and let $f^{n}$ denote the map $f$ repeated $n$-times. We denote by $f^{n} R$ the bi- $R$-algebra $R$, having the structure of an $R$-algebra from the left by $f^{n}$ and from the right by identity. For any $R$-module $M, F_{R}^{n}(M)$ will stand for $M \otimes_{R} f^{n} R$ and $f^{n} N$ will denote the module $N$ viewed as an $R$-module via $f^{n}[P-S]$. Avramov and Miller proved the following:

Theorem $(\underline{\mathrm{A}-\mathrm{M}}])$. Let $M$ be a finitely generated module over a local complete intersection ring $(R, m, k)$. If for some $i>0$ and for some $n>0, \operatorname{Tor}_{i}^{R}\left(M,{ }^{n} R\right)=$ 0 , then $M$ is of finite projective dimension.

Their proof used the notion of "complexity" and several related theorems. Here, we intend to provide a much simpler proof which does not use "complexity" at all. The only well-known theorems we exploit are the following: the theorem on flatness of the frobenius due to Kunz [K, 3.3] and Herzog's theorem on characterization of finite projective dimension [H, 3.1].

Historically, work on such problems started in 1969 with Kunz's theorem [K] 3.3] on equivalence of flatness of $f^{n}$ for all $n \geq 1$ with the regularity of the local ring $R$. Next, Peskine and Szpiro established the following $[\mathrm{P}-\mathrm{S}, 1.7]$ : If a finitely generated $R$-module $M$ has finite projective dimension over $R, \operatorname{Tor}_{i}^{R}\left(M, f_{R}^{n}\right)=$ 0 for all $i, n \geq 1$. Then Herzog [H, 3.1] proved the converse: If $M$ is finitely generated and $\operatorname{Tor}_{i}^{R}\left(M, f_{R}^{n}\right)=0$ for all $i>1$ and infinitely many $n, M$ has finite projective dimension over $R$. The theorem of Avramov and Miller stated above

Received by the editors June 18, 2001 and, in revised form, September 3, 2001.

2000 Mathematics Subject Classification. Primary 13C14, 13C40, 13D05, 13D40, $13 \mathrm{H} 10$.

Key words and phrases. Complete intersection, finite projective dimension, flatness, Frobenius, Tor.

This research was partially supported by an NSF grant. 
is a much desired extension of Herzog's theorem to complete intersections. For modules $M$ of finite length over a complete interection this author showed [D, 1.9] that $\ell\left(F_{R}^{n}(M)\right) \geq p^{n d} \ell(M)$ where $d$ denotes dimension of $R$ and sign of equility holds when $M$ is of finite projective dimension; in [M, 1.1] Miller proved the converse and deduced the following: If for some $i>0, \lim _{n \rightarrow \infty} \ell\left(\operatorname{Tor}_{i}^{R}\left(M, f_{R}^{n}\right)\right) / p^{n d}=0$, then $M$ has finite projective dimension.

We accomplish our proof in the following steps.

Step 0. Without any loss of generality, we can assume that $R$ is complete and $R=S / \underline{x}$ where $S$ is a complete regular local ring of characteristic $p>0$ and $\underline{x}=\left(x_{1}, \ldots, x_{r}\right)$ is the ideal generated by an $S$-sequence $x_{1}, \ldots, x_{r}$. Let $d$ be dimension of $R$ (henceforth $\operatorname{dim}$ ); then $r+d=\operatorname{dim} S$. We know by Kunz's theorem that $f^{n}: S \rightarrow S: f^{n}(x)=x^{p^{n}}$ is a flat map $\forall n>0$.

Step 1. Since $S \stackrel{f^{n} S}{\longrightarrow} S$ is flat, $S / \underline{x} \stackrel{\tilde{f}^{n}}{\longrightarrow} S / \underline{x}^{p^{n}}$ is flat (base change). Hence $R \stackrel{f^{n} R}{\longrightarrow}$ $R$ can be factored as

$$
R=S / \underline{x} \stackrel{\tilde{f}^{n}}{\longrightarrow} S / \underline{x}^{p^{n}} \stackrel{\eta_{n}}{\longrightarrow} R=S / \underline{x}
$$

where $\eta_{n}$ is the natural surjection.

Thus $f^{n} R=\eta_{n} \cdot \tilde{f}^{n}$.

Let $M$ be a finitely generated $R$-module. Consider an exact sequence (a presentation of $M$ )

$$
R^{t_{1}} \stackrel{\phi}{\rightarrow} R^{t_{0}} \rightarrow M \rightarrow 0
$$

Apply $\otimes f^{n} S$ and obtain an exact sequence

$$
\left(S / \underline{x}^{p^{n}}\right)^{t_{1}} \stackrel{\phi^{\left[p^{n}\right]}}{\longrightarrow}\left(S / \underline{x}^{p^{n}}\right)^{t_{0}} \rightarrow F_{S}^{n}(M) \rightarrow 0 .
$$

(1) and (2) imply that

$$
M \otimes_{R} \tilde{f}^{n}\left(S / \underline{x}^{p^{n}}\right) \simeq F_{S}^{n}(M) .
$$

Since $\tilde{f}^{n}$ is flat, we have

$$
\operatorname{Tor}_{i}^{R}\left(M, f^{n} R\right)=\operatorname{Tor}_{i}^{R_{n}}\left(F_{S}^{n}(M), S / \underline{x}\right),
$$

where $R_{n}=S / \underline{x}^{p^{n}}$

Step 2. We want to show that $\operatorname{Tor}_{1}^{R}\left(M, f^{n} R\right)=0$ implies that $\operatorname{Tor}_{i}^{R}\left(M, f^{n} R\right)=0$ for $i \geq 1$. It is enough to show that $\operatorname{Tor}_{2}^{R}\left(M, f^{n} R\right)=0$. By flatness of $\tilde{f}^{n}$ we will be done by showing that

$$
\operatorname{Tor}_{1}^{R_{n}}\left(F_{S}^{n}(M), S / \underline{x}\right)=0 \quad \text { implies } \quad \operatorname{Tor}_{2}^{R_{n}}\left(F_{S}^{n}(M), S / \underline{x}\right)=0 .
$$

We know $S / \underline{x}^{p^{n}}$ has a filtration such that successive quotients are isomorphic to $S / \underline{x}$. We have the following exact sequences:

$$
\begin{gathered}
0 \rightarrow K_{1} \rightarrow S / \underline{x}^{p^{n}} \rightarrow S / \underline{x} \rightarrow 0, \\
0 \rightarrow K_{2} \rightarrow K_{1} \rightarrow S / \underline{x} \rightarrow 0, \\
0 \rightarrow K_{t_{n}} \rightarrow K_{t_{n-1}} \rightarrow S / \underline{x} \rightarrow 0
\end{gathered}
$$


where $K_{t_{n}}=S / \underline{x}$. Since $\operatorname{Tor}_{1}^{R_{n}}\left(F_{S}^{n}(M), S / \underline{x}\right)=0$, we obtain by going up along the above exact sequences successively that $\operatorname{Tor}_{1}^{R_{n}}\left(F_{S}^{n}(M), K_{1}\right)=0$. This implies that $\operatorname{Tor}_{2}^{R_{n}}\left(F_{S}^{n}(M), S / \underline{x}\right)=0$. Hence $\operatorname{Tor}_{2}^{R}\left(M, f^{n} R\right)=0$.

Step 3. We want to show that $\operatorname{Tor}_{1}^{R}\left(M,{ }^{n} R\right)=0$ implies that $\operatorname{Tor}_{1}^{R}\left(M,{ }^{n+1} R\right)=$ 0 . Hence, we need to show that $\operatorname{Tor}_{1}^{R_{n+1}}\left(F_{S}^{n+1}(M), S / \underline{x}\right)=0$, provided that $\operatorname{Tor}_{1}^{R_{n}}\left(F_{S}^{n}(M), S / \underline{x}\right)=0$. Recall from Step 2 that since $\operatorname{Tor}_{1}^{R_{n}}\left(F_{S}^{n}(M), S / \underline{x}\right)=$ $0, \operatorname{Tor}_{i}^{R_{n}}\left(F_{S}^{n}(M), S / \underline{x}\right)=0$ for $i \geq 1$ and from Step 1 that $R_{n} \stackrel{\tilde{f}}{\rightarrow} R_{n+1}$ is flat. Hence (tensoring the above equation by ${ }^{f} R_{n+1}$ ),

$$
\operatorname{Tor}_{i}^{R_{n+1}}\left(F_{S}^{n+1}(M), S / \underline{x}^{p}\right)=0 \quad \text { for } i \geq 1 .
$$

Consider the short exact sequences

$$
0 \rightarrow S /\left(x_{1}, x_{2}^{p}, \ldots, x_{r}^{p}\right) \rightarrow S /\left(x_{1}^{p+1}, x_{2}^{p}, \ldots, x_{n}^{p}\right) \rightarrow S / \underline{x}^{p} \rightarrow 0
$$

and

$$
0 \rightarrow S / \underline{x}^{p} \rightarrow S /\left(x_{1}^{p+1}, x_{2}^{p}, \ldots, x_{r}^{p}\right) \rightarrow S /\left(x_{1}, x_{2}^{p}, \ldots, x_{r}^{p}\right) \rightarrow 0 .
$$

Applying (5), from (6) we obtain

$$
\begin{aligned}
& \operatorname{Tor}_{i}^{R_{n+1}}\left(F_{S}^{n+1}(M), S /\left(x_{1}, x_{2}^{p}, \ldots, x_{r}^{p}\right)\right) \\
& \quad \simeq \operatorname{Tor}_{i}^{R_{n+1}}\left(F_{S}^{n+1}(M), S /\left(x_{1}^{p+1}, x_{2}^{p}, \ldots, x_{r}^{p}\right)\right) \quad \text { for } i \geq 1,
\end{aligned}
$$

and applying (5), from (7) we obtain

$$
\begin{aligned}
& \operatorname{Tor}_{i}^{R_{n+1}}\left(F_{S}^{n+1}(M), S /\left(x_{1}^{p+1}, x_{2}^{p}, \ldots, x_{r}^{p}\right)\right) \\
& \quad \hookrightarrow \operatorname{Tor}_{i}^{R_{n+1}}\left(F_{S}^{n+1}(M), S /\left(x_{1}, x_{2}^{p}, \ldots, x_{r}^{p}\right)\right) \text { for } i \geq 1
\end{aligned}
$$

which is an isomorphism for $i \geq 2$ and an injection for $i=1$.

Note that the composition of the two maps (8) and (9) is the one induced by mutiplication by $x_{1}^{p}$.

Combining (6), (7), (8) and (9) we observe that

$$
S /\left(x_{1}, x_{2}^{p}, \ldots, x_{r}^{p}\right) \stackrel{x_{1}^{p}}{\longrightarrow} S /\left(x_{1}, x_{2}^{p}, \ldots, x_{r}^{p}\right)
$$

is the 0-map and it induces an injection between

$$
\operatorname{Tor}_{i}^{R_{n+1}}\left(F_{S}^{n+1}(M), S /\left(x_{1}, x_{2}^{p}, \ldots, x_{r}^{p}\right)\right) \hookrightarrow \operatorname{Tor}_{i}^{R_{n+1}}\left(F_{S}^{n+1}(M), S /\left(x_{1}, x_{2}^{p}, \ldots, x_{r}^{p}\right)\right)
$$

for $i \geq 1$.

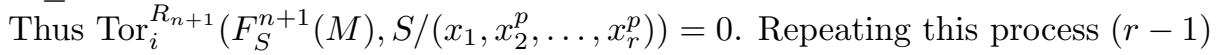
times we obtain

$$
\operatorname{Tor}_{i}^{R_{n+1}}\left(F_{S}^{n+1}(M), S /(\underline{x})=0 \quad \text { for } i \geq 1 .\right.
$$

Step 4. Steps 1, 2, 3 and the well-known theorem due to Herzog [H, 3.1] prove the assertion stated in the theorem at the beginning.

Remark. With notation as above, over a complete intersection $R=S / \underline{x}$, for a finitely generated module $M$, we have $\operatorname{Pd}_{R} M<\infty$ if and only if $\operatorname{Pd}_{R_{n}} F_{S}^{n}(M)<\infty$.

The proof follows easily from Step 1. 


\section{REFERENCES}

[A-M] L. Avramov and C. Miller, Frobenius powers of complete intersections, Math. Research Letters 8, nos. 1 and 2, 2001, 225-232. MR 2002b:13022

[D] S. P. Dutta, Frobenius and Multiplicities, J. Algebra 85 (1983), 424-448. MR 85f:13022

[H] J. Herzog, Ringe de Charakteristik p und Frobeniusfunktoren, Math Z. 140 (1974), 67-78. MR 50:4569

[K] E. Kunz, Characterization of regular local rings for characteristic p, Amer. J. Math. 91 (1969), 772-784. MR 40:5609

[M] C. Miller, A Frobenius characterization of finite projective dimension over complete intersections, Math. Z. 233 (2000), 127-136. MR 2001a:13037

[P-S] C. Peskine and L. Szpiro, Dimension projective finie et cohomologie locale, I.H.E.S. Publ. Math. 42 (1973), 47-119. MR 51:10330

Department of Mathematics, University of Illinois, 1409 West Green Street, UrBANA, ILLINOIS 61801

E-mail address: dutta@math.uiuc.edu 